\title{
Phytohormones, plant growth regulators and signaling molecules: cross-talk and stress responses
}

\author{
Aryadeep Roychoudhury ${ }^{1}$ Tariq Aftab ${ }^{2}$
}

Received: 15 July 2021 / Accepted: 15 July 2021 / Published online: 17 July 2021

(c) The Author(s), under exclusive licence to Springer-Verlag GmbH Germany, part of Springer Nature 2021

\begin{abstract}
Key message Interactions of phytohormone signaling pathways and their crosstalk with the different intermediates of cell signaling cascades regulate the molecular stress responses in plants.
\end{abstract}

Plants, being sessile organisms, are constantly exposed to a wide range of environmental variations, including perturbations from salinity, drought, temperature, irradiance (including UV radiation), heavy metal/metalloid exposure, and bioaccumulation, and increasing $\mathrm{CO}_{2}$ concentration. Some of these factors are stressful to the plants and exacerbated by unprecedented climate change. Biotic challenges that plants face include herbivores and microbial pathogens, including bacteria, fungi, viruses, and nematodes. Such stresses, either alone or in combination, evoke detrimental consequences to growth, productivity, and yield. Severe oxidative damages may occur, which disrupt the structural integrity of plant biomolecules, thereby collapsing the vital physiological processes and redox homeostatic balance (Roychoudhury et al. 2008). In an attempt to counteract such critical events, plants employ a variety of biochemical pathways, which activate a wide array of phytohormones, growth regulators, and signaling molecules, the interaction or cross-talk of which helps in plant protection. Given the complexities, a complete understanding of mechanisms must rely on studying the elaborate interplay of phytohormones and signaling molecules.

For a long time, abscisic acid (ABA) was considered to be the major phytohormone exclusively connected with stomatal regulation and plant stress responses. With the

Communicated by high priority .

Aryadeep Roychoudhury

aryadeep.rc@gmail.com

1 Post Graduate Department of Biotechnology, St. Xavier's College (Autonomous), 30, Mother Teresa Sarani, Kolkata 700016, West Bengal, India

2 Department of Botany, Aligarh Muslim University, Aligarh, Uttar Pradesh 202002, India onset of stress, the endogenous ABA level increases, which causes stomatal closure through slow-acting sustained (S-type) channels, i.e., the slow anion channel-associated 1 (SLAC1) that triggers membrane depolarization (JoshiSaha et al. 2011; Negi et al. 2008). Enhanced reactive oxygen species (ROS) production in guard cells affected by ABA accumulation also creates a positive feedback loop to mediate stomatal closure, which limits water loss from the leaves (Mittler and Blumwald 2015). In addition to its role in regulating stomatal aperture, ABA induces the expression of many genes whose products are important for stress responses and tolerance. Such products include osmoprotectant-related enzymes and late embryogenesis abundant proteins (Roychoudhury et al. 2013). With further advancement in research and discovery of emerging signaling molecules, the concept of ABA, being the only major 'stress phytohormone' has radically changed. Almost all the other phytohormones, such as auxins, gibberellins, cytokinins, and ethylene are equally capable of regulating stress responses by exerting their functions in their own ways so that such a line of demarcation among the phytohormones in terms of the stress response is slowly fading away. The emerging evidence for the interaction of the gibberellic acid (GA)-signaling molecule, DELLA, with components of the signaling pathway for the stress hormone, jasmonic acid, suggested clear involvement of GA in stress responses (Colebrook et al. 2014). The effects of cytokinin on plant xenobiotic resistance, and its interactions with drought, salinity, temperature, and light signals, are also increasingly being recognized (Pavlu et al. 2018). The roles of ethylene in tolerance against salinity, $\mathrm{K}^{+}$ deficiency and Fe deficiency, as well as against infections by Alternaria alternata are reported (Khan et al. 2017). These observations justify that abiotic stress tolerance and biotic stress resistance in plants are well-orchestrated by elaborate 
interactions among different phytohormones and signaling molecules that mutually influence the levels and functions of each other.

Newly-discovered signaling functions of salicylic acid, jasmonic acid, nitric oxide, hydrogen sulfide, melatonin, as well as others, indicate coordination as synergistic or antagonistic functions. Signaling of the phenolic hormone, salicylic acid (SA) is integrated with ABA signaling in guard cells. SA activates peroxidase-mediated ROS signal that is integrated into $\mathrm{Ca}^{2+} /$ calcium dependent protein kinasesmediated ABA signaling branch (Prodhan et al. 2018). The role of exogenous SA in alleviating bacterial, fungal, and viral diseases, heavy metal toxicity, the toxicity of essential micronutrients, and salt stress has been extensively reviewed (Wani et al. 2017). The CORONATINE INSENSITIVE 1 (COI1) protein, JASMONATE ZIM domain protein (JAZ), and MYC constitute the core signal transduction mechanism of jasmonic acid (JA) signaling and have proven to be the intersection of signal transduction pathways with other hormones like auxin, ethylene, ABA, SA, brassinosteroids, and GA under various forms of stresses, as well as defense against hemibiotrophic pathogens such as Magnaporthe oryzae and Pseudomonas syringae (Yang et al. 2019). Application of exogenous ubiquitous gasotransmitter, nitric oxide (NO), can alleviate the negative effects of stress in plants, improve antioxidant activity in most plant species and regulate specific checkpoints to control the switch between plant development and stress, via post-translational protein modifications, viz., S-nitrosylation of cysteine residues and metals, and nitration of tyrosine residues (Sanchez-Vicente et al. 2019). The rapid production of endogenous hydrogen sulfide in many species can also be triggered by numerous stresses, so that it appears as a signaling molecule for cross-adaptation to pathogens, salt, drought, heavy metals, cold, heat, and flooding stress by enhancing antioxidant system activity, accumulating osmolytes, synthesizing heat shock proteins, as well as maintaining ion and nutrient balance ( $\mathrm{Li}$ et al. 2016). The intrinsic nature of melatonin to act as a nodal antioxidant upon exogenous application to plants has been recently shown in several plant species and under different types of sub-optimal conditions. The functions of melatonin in regulating the homeostasis of major phytohormones like auxins, GA, and ABA under different stresses are also evident from recent investigations (Banerjee and Roychoudhury 2019). Calcium acts as the second messenger involved in the signaling pathways of several phytohormones. Cytosolic free calcium is central to the stress response in plants, concurrent with the opening of the plasma membrane and endomembrane $\mathrm{Ca}^{2+}$-permeable channels and terminated by the activity of $\mathrm{Ca}^{2+}$ efflux transporters in those membranes, plus $\mathrm{Ca}^{2+}$-binding proteins, to restore the resting cytoplasmic concentration of 100-200 nM. Calcium signaling during nutrient deprivation is also an emerging field of research
(Wilkins et al. 2016). Taken altogether, elucidation of the intricate mechanism of interactions of phytohormone signaling pathways and their integration with the intermediates of other signaling cascades is quintessential to properly understand the molecular regulation of plant stress response.

Keeping in view this elaborate and intricate cross-talk between phytohormones and signaling molecules, the first volume of the current special issue is intended to decipher the interaction and cross-talk among a large number of plant growth regulators and signaling molecules that regulate stress response in plants, based on both biochemical and molecular approaches. This volume includes fifteen research and review articles, encompassing diverse growth regulators and signaling molecules, which were contributed by esteemed authors, expert in their relevant fields. The research presented pushes the field ahead in a coherent collection of timely topical papers.

Author contribution Dr. Aryadeep Roychoudhury drafted the Editorial. Dr. Tariq Aftab assisted him in collecting the references and providing necessary suggestions.

\section{References}

Banerjee A, Roychoudhury A (2019) Melatonin application reduces fluoride uptake and toxicity in rice seedlings by altering abscisic acid, gibberellin, auxin and antioxidant homeostasis. Plant Physiol Biochem 145:164-173

Colebrook EH, Thomas SG, Phillips AL, Hedden P (2014) The role of gibberellin signalling in plant responses to abiotic stress. J Exp Biol 217:67-75

Joshi-Saha A, Valon C, Leung J (2011) A brand new START: abscisic acid perception and transduction in the guard cell. Sci Signal 4:re4

Khan NA, Khan MIR, Ferrante A, Poor P (2017) Editorial: ethylene: a key regulatory molecule in plants. Front Plant Sci 8:1782

Li Z-G, Min X, Zhou Z-H (2016) Hydrogen sulfide: a signal molecule in plant cross-adaptation. Front Plant Sci 7:1621

Mittler R, Blumwald E (2015) The roles of ROS and ABA in systemic acquired acclimation. Plant Cell 27:64-70

Negi J, Matsuda O, Nagasawa T, Oba Y, Takahashi H, Kawai-Yamada $\mathrm{M}$ et al (2008) CO2 regulator SLAC1 and its homologues are essential for anion homeostasis in plant cells. Nature 452:483-486

Pavlů J, Novák J, Koukalová V, Luklová M, Brzobohatý B, Černý M (2018) Cytokinin at the crossroads of abiotic stress signalling pathways. Int J Mol Sci 19:2450

Prodhan MY, Munemasa S, Nahar MN-E-N, Nakamura Y, Murata Y (2018) Guard cell salicylic acid signaling is integrated into abscisic acid signaling via the $\mathrm{Ca}^{2+} / \mathrm{CPK}$-dependent pathway. Plant Physiol 178:441-450

Roychoudhury A, Basu S, Sarkar SN, Sengupta DN (2008) Comparative physiological and molecular responses of a common aromatic indica rice cultivar to high salinity with non-aromatic indica rice cultivars. Plant Cell Rep 27:1395-1410

Roychoudhury A, Paul S, Basu S (2013) Cross-talk between abscisic acid-dependent and abscisic acid-independent pathways during abiotic stress. Plant Cell Rep 32:985-1006 
Sánchez-Vicente I, Fernández-Espinosa MG, Lorenzo O (2019) Nitric oxide molecular targets: reprogramming plant development upon stress. J Exp Bot 70:4441-4460

Wani AB, Chadar H, Wani AH et al (2017) Salicylic acid to decrease plant stress. Environ Chem Lett 15:101-123

Wilkins KA, Matthus E, Swarbreck SM, Davies JM (2016) Calciummediated abiotic stress signaling in roots. Front Plant Sci 7:1296

Yang J, Duan G, Li C, Liu L, Han G, Zhang Y, Wang C (2019) The crosstalks between jasmonic acid and other plant hormone signaling highlight the involvement of jasmonic acid as a core component in plant response to biotic and abiotic stresses. Front Plant Sci 10:1349

Publisher's Note Springer Nature remains neutral with regard to jurisdictional claims in published maps and institutional affiliations. 